\title{
A Software Architecture for Collaborative Training in Virtual Worlds: F-16 Airplane Engine Maintenance
}

\author{
Benjamim Fonseca ${ }^{1}$, Hugo Paredes ${ }^{1}$, Lt. Jorge Rafael ${ }^{2}$, Leonel Morgado ${ }^{1}$, \\ and Paulo Martins ${ }^{1}$ \\ 1 GECAD/UTAD - University of Trás-os-Montes e Alto Douro, \\ Quinta de Prados, Apartado 1013, Vila Real, Portugal \\ \{benjaf, hparedes, leonelm, pmartins\}@utad.pt \\ 2 Força Aérea Portuguesa - Base Aérea $\mathrm{N}^{\circ} 5$, \\ Serra de Porto de Urso, Monte Real - Leiria, Portugal \\ tenjorgerafael@gmail.com
}

\begin{abstract}
The maintenance of military aircraft is complex and exhaustive, requiring an accurate training program. This process is not fault tolerant and requires certification renewal periodically. Furthermore, the process involves many professionals and resources, requiring phases of maintenance and verification of the tasks. Cooperation between professionals in the overall process is essential and requires strong team coordination. It is a highly costly process, since aircrafts are scarce and their readiness is essential for missions, and it requires a scheduling effort between all team members and aircrafts. The availability of tools that allow intensive training without aircraft presence is an asset to the maintenance squadrons. Virtual worlds have simulation and collaboration capabilities to implement this process. This paper presents a software architecture developed for training engine maintenance squadrons for certification, using virtual worlds platforms. This architecture is being tested in cooperation with the Portuguese Air Force and an engine maintenance squadron of F-16 aircrafts.
\end{abstract}

Keywords: Cooperation processes, task coordination, virtual worlds, aircraft engine maintenance.

\section{Introduction}

The maintenance of military aircrafts is a complex, rigorous, delicate and exhaustive process requiring a very accurate training program and a renewal of certification in regular periods of time. The process involves a large number of physical resources and several people, requiring phases of maintenance and verification of the performed tasks. Cooperation between team members in both phases is essential to the success of the overall process. Moreover there is a special concern regarding the coordination of every team task. Concerning the material resources needed, the process involves high costs, since aircrafts are scarce and 
their readiness is essential for participation in air force missions. This implies a scheduling effort that requires the simultaneous availability of maintenance crews and aircrafts. In this context, the availability of a software tool which allows a more intensive and frequent training of the team without the physical presence of a real aircraft is an asset to the maintenance squadrons of the Air Force. Virtual worlds have characteristics, including simulation and collaboration capabilities and the 3D environment, which are adequate to implement the process in a virtual environment.

This paper presents a software architecture developed for training engine maintenance squadrons in virtual worlds, in order to perform the required preparation for the certifications required regularly in a virtual environment. This architecture is being tested in a real case that involves collaboration with the Portuguese Air Force and an engine maintenance squadron of F-16 aircrafts. The current implementation allows mechanics teams to train the operations of attaching and detaching an engine into the F-16 aircraft, as specified in its technical orders. For every step in the maintenance process, the user is allowed to take the initiative to grab a tool and operate it, but the system ensures that he does it in the right sequence and acting coordinated with other team members. However, the system was designed to allow the future introduction of error margins, lack of coordination, and erroneous procedures, in order to observe the consequences of incorrect operation. The architecture separates the user interface, built in Open Simulator, from the control system, implemented externally to ensure modularity, evolution and platform independence.

Section 2 presents some background concepts of virtual worlds and its use for educational, training and simulation purposes. Section 3 describes the methodology used in the requirement analysis and section 4 presents the overall system architecture. Finally, some final remarks are presented in section 5 , pointing out some conclusions and future directions.

\section{Background}

Virtual worlds environments are digital representations of real or imaginary scenarios that intend to simulate reality or compliment it and enable multiple users to be present and communicate with each other 8. Early virtual environments worlds were based on textual descriptions and interactions, and appeared in 1979 as Multi-User Dungeons (MUD) 5]. Subsequently, the web introduced the concept of the concept evolved into illustrated virtual worlds (known as "graphical MUDs") and 2D/3D immersive virtual environments worlds. The last decade witnessed the emergence of the latter variety, graphic-intensive 3D multiuser virtual worlds that enable people to conduct several distinct activities, ranging from entertainment and socialization, to professional activities like meetings, marketing campaigns, collaborative learning and education/training [1]4]8]3]6]. This has been particularly noticeable since $2007 / 2008$ [3].

Nakasone [9] presented Astrosim, a collaborative environment built in Second Life, where users can visualize astronomy simulations. Creutzfeldt 2] presents 
the use of virtual worlds for the collaborative training of medical students in cardiopulmonary resuscitation. Other uses for medical education exist, such as [1311. Wyld [14 presents other simulated training environments, discussing its importance for several leading-edge organizations and the adequacy of such approach for the digital natives generation.

Military training is a critical issue for the success of military operations, either for tactical and fighting activities, as for technical operations such as aircraft piloting or maintenance. Simulation plays an important role here, and its application for technical operations is well-know, with several special purpose simulators that enable a single user to train specific technical or dangerous tasks. There are also some games that are used by multiple users for training fighting and tactical activities [12 10], but the area of multi-user tactical training simulations is in its infancy.

\section{Approach}

In the Portuguese Air Force (FAP, Portuguese-language acronym), the basic training of the Air Materiel Mechanics is performed at the Air Force Centre for Military and Technical Training. After this training, soldiers are assigned to the units where they receive specific training, as is the case of the Air Base 5, near Monte Real/Leiria, Portugal, where F-16 aircrafts of the FAP are based. In this unit, the training of Air Material Mechanics integrates theoretical stages and also practical phases, which must necessarily be carried out on real components, in order to receive certification for the maintenance of that aircraft engine. Thus, the practical component of the training requires that planes and/or engines involved in it are available for that purpose, which makes this training particularly expensive. For this reason, the adoption of a 3D virtual learning environment for training contributes to increase the efficiency of this training phase with real components, because that enables trainees to perform simulated maintenance without the involvement of physical resources.

The aircraft maintenance process involves several phases, and, in order to evaluate the usage of $3 \mathrm{D}$ virtual learning environment for training, one phase of the process that reflects the needs of the FAP was chosen. Moreover, from a technical perspective, a phase that reflects the requirements for cooperation between those involved in maintenance, as well as the manipulation of large and expensive objects was selected. Regarding thus, the phase of maintenance chosen, in collaboration with the FAP was the installation of a Pratt \& Whitney F100.PW.220/220E engine in a F-16 aircraft.

The chosen methodology for the problem analysis was based on the aeronautic training process following, necessarily, a different set of execution tasks, which may be interdependent or not. In aircraft maintenance mechanics, these processes are specified by the manufacturers, through documents called Technical Orders (TO), which describe the execution tasks of the various phases in text and diagrams. Thus, the TO associated with the engine [7] show all actions to complete a particular process in the engine. To facilitate the analysis process, as 
a cooperative process, that information was complemented with video recordings and images of the actual processes and transformed into a cooperation script.

The developed cooperation script was divided in three main phases: (1) preinstallation, which involves the verification processes: engine conformity; security conditions; toolbox; and availability of the necessary part to engine installation. In this phase, the current TO should be consulted; (2) installation of engine in the aircraft, which includes its transfer for the transportation vehicle to the aircraft following the order defined in the TO; (3) post-installation, where the engine installation is supervised, the toolbox is checked to avoid foreign object damage and the engine is tested. The pre and post installation phases require only the participation of one maintenance mechanic and there is only basic requirements for cooperation in order to, respectively, prepare and finalize the engine installation task. On the other hand, the engine installation phase needs the cooperation of four mechanics in order to perform the tasks defined in the TO. At this point, the main problem is the tasks' division by the mechanics, since this is not defined in the TO. Therefore it was necessary to observe the teams in real engine installation processes in order to define clearly the tasks of each team member. Thus, the script was divided into four sub-scripts, corresponding to the tasks of each mechanic. Furthermore, common trunk was also included, in which the interdependencies are identified and correlated to the sub-scripts.

\section{Architecture}

The software architecture developed for the training of engine maintenance squadrons has as main goals: (1) multi-layer: ensuring the independence of the coordination and interaction mechanisms made available by the 3D environment, in order to ensure the separation between the interface and the decision layer of the architecture; (2) evolutive: allowing changes from the technical evolution of the engine maintenance processes, and concerning the changes that are introduced by the vendors in the engine TO; (3) multi-platform: defining a generic model that can be implemented in several virtual worlds.

Through the objectives set out for the architecture, in the following subsections its specification is presented and an implementation based on the OpenSim virtual worlds technology is proposed, which is being tested in the maintenance of F-16 aircraft by the FAP.

\subsection{Specification}

The specification of the architecture was based on the cooperation script developed in a phase of analysis and previously presented. The cooperation script is a text document that outlines the process of the engine installation on the aircraft, and is divided, as above mentioned, in four sub-scripts and a transverse component that specifies the interrelationships between the tasks of each actor in the process. The document identifies 36 tasks that require full alignment between the cell and the engine of the aircraft, as well as their readiness for the 
installation phase. The first 19 execution tasks, summarized in Table 1, refer to the motor coupling to the cell, while the remaining tasks correspond to connecting operations that are performed on the right (6) and left (8) side of the aircraft, and the finalization of the process with connection of the master fuel to the cockpit, initialization of the engine EDU and installation of the fuselage.

Table 1. Main execution tasks for engine installation

\begin{tabular}{llc}
\hline \hline task & task & TO \\
number & description & $2-93$ \\
\hline \hline 1 & Installation of the engine mount to raise the engine \\
2 & Engine raise & $2-93$ \\
3 & Couple engine to the aircraft & $2-93$ \\
4 & Perfect alignment of the upper engine mount with the fuselage rail $2-95$ \\
5 & Fasten and break of the trailer to the fuselage & $2-95$ \\
6 & Adjustment of the trailer to transfer engine weight & $2-95$ \\
7 & Support the engine on the fuselage rail & $2-97$ \\
8 & Enter the engine in perfect alignment with the fuselage & $2-100$ \\
9 & Align with the trust pin connections & $2-100$ \\
10 & Enter the trust pins using the connection doors & $2-100$ \\
11 & Finish the back & $2-100$ \\
12 & Check perfect alignment of the trust pin with the clamp half & $2-101$ \\
13 & Tighten up the clamp half with a ring (nut) to finish baking & $2-101$ \\
14 & Finish installing the trust pin with an inspection & $2-103$ \\
15 & Check the SEAL, from the air entrance & $2-101$ \\
16 & Remove the trailer & $2-111$ \\
17 & Remove the back engine adapter & $2-111$ \\
18 & Lighten/download support fuselage & $2-113$ \\
19 & Completely remove the trailer & \\
\hline \hline
\end{tabular}

The adaptation of the cooperation script to the specification of the architecture to support the training system went through its translation to UML language. Thus, based on the script, the actors of the system and the main use cases were identified and the main operations were specified using use cases diagrams. The refinement of this specification was performed defining state and activities diagrams for the whole process. However, during the specification process, two subsystems were identified: the interaction and the decision subsystems. It was also found that the script was referring to the interaction subsystem, so there was a need for the specification of the mechanisms of decision and communication between the subsystems.

In terms of specification of the decision subsystem, a workflow has been specified based on the TO associated with the engine installation. Regarding the mechanisms of communication and interaction a generic interaction system has been defined, shown in Figure 1. The specification of this generic system defines that when an avatar touches an object in the virtual world an event that is communicated to the decision system is fired. Based on the received event, the 


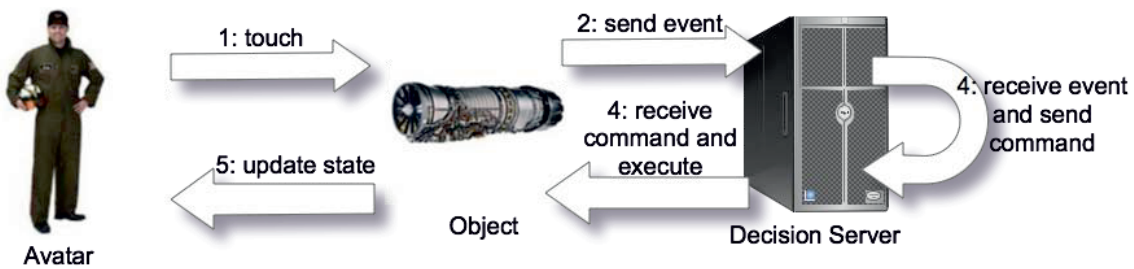

Fig. 1. Generic interaction system

decision system replies to the object sending a command to be executed, which is reflected in the state of the avatar. The architecture is then divided into two logical components: the virtual world and the decision system. The implementation of this system, as well as discussion of technological options for its development, are presented in the next subsection.

\subsection{Implementation}

The software architecture was implemented as a client-server architecture. The client side is delegated to the virtual world client, and the server side includes the virtual world server and the decision server.

The choice for the development of the environment of the virtual world was made based on the requirements of the FAP. The environment should be closed to a certain number of users which are allowed to perform training of engine maintenance, so the virtual world server should be private and the creation of avatars must be controlled. Moreover, the virtual world should allow users to model their own objects and must be programmable in order to implement the generic interaction system previously presented. Based on this requirement the virtual world server chosen was the OpenSimulator, "an open source multiplatform, multi-user 3D application server that can be used to create a virtual worlds" (http://opensimulator.org).

In the virtual world was modeled an hangar environment with similar characteristics to the FAP hangar in Air Base 5 (Figure 2). The environment modeling also included the F-16 aircrafts, their engines and all components and materials required for the execution of the engine installation. Each object had a special script associated with the touch event, developed in Linden Scripting Language (LSL), to send information about the environment to the decision server. This communication is performed using HTTP requests and encoding in XML language, allowing a standard communication between the two system modules.

The decision server is implemented in ASP.Net platform and is based on the session facade design pattern, defining a single point of entry for the decision module. This module acts based on the implementation of a balanced decision tree that keeps the system state on each step of the engine installation process. Moreover, the system also keeps track on the current state of each object of the 


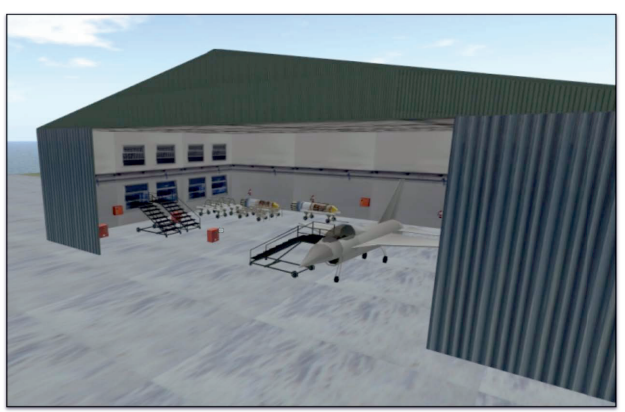

Fig. 2. Virtual representation of FAP hangar in Air Base 5

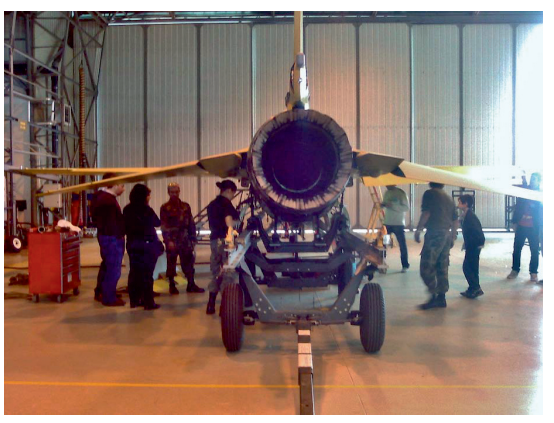

Fig. 3. Real engine installation situation at FAP Air Base 5

system in order to infer at each system state what are the available tasks to be performed by the mechanicals in the training process.

\section{$5 \quad$ Final Remarks}

This paper presented a software architecture that uses virtual worlds for training F-16 engine maintenance squadrons, which are subject to regular certification. This simulator enables the maintenance crew training to be independent of aircraft availability, facilitating its occurrence on a regular basis to compliment the certification and training under real situation, which must still occur.

The study of the technical orders of the PRATT \& WHITNEY F100.PW.220/ $220 \mathrm{E}$ engine was a crucial step in understanding the requirements of the system in terms of tools and parts manipulation, as well as on the correct sequence of activities to be carried out. The direct observation of the daily work of the F-16 maintenance squadron was also important, as it enabled a better understanding of the team coordination and of a few clues that are described in the TO.

The system adopted a multi-level architecture in order to cope with the independence of interaction and control mechanisms and empowering further updates. It was also important to adopt a hierarchical state machine because the whole process encompasses both system and individual object states.

The prototype implementation deals currently with the attaching of the F-16 engine to the aircraft without the possibility of errors or failures. The further evolution of the system will contemplate the allowance of errors, the detaching process and the separation in blocks and parts. Another important feature to be introduced is the autonomous operation of avatars, enabling training in the absence of one or more team members.

Acknowledgements. We would like to thank the collaboration of Portuguese Air Force, Air Force Base 5 - Monte Real, Leiria, Portugal, for all the support they provided for the development of this project. We would also like to thanks 
the undergraduate and graduate students of the University of Trás-os-Montes e Alto Douro, which were involved in this process, for their support in the implementation of the system.

\section{References}

1. Adobor, H., Daneshfar, A.: Management simulations: determining their effectiveness. The Journal of Management Development 25(2), 151-168 (2006)

2. Creutzfeldt, J., Hedman, L., Medin, C., Heinrichs, W.L., Felländer-Tsai, L.: Exploring virtual worlds for scenario-based repeated team training of cardiopulmonary resuscitation in medical students. Journal of Medical Internet Research 12(3), e38 (2010); PMID: 20813717

3. Dalgarno, B., Lee, M.J.W., Carlson, L., Gregory, S., Tynan, B.: An australian and new zealand scoping study on the use of $3 \mathrm{~d}$ immersive virtual worlds in higher education. Australasian Journal of Educational Technology 27(1), 1-15 (2011)

4. Esteves, M., Fonseca, B., Morgado, L., Martins, P.: Improving teaching and learning of computer programming through the use of the second life virtual world. British Journal of Educational Technology (2010)

5. Fanderclai, T.L.: Muds in education: New environments, new pedagogies. Computer-Mediated Communication Magazine 2(1), 8 (1995)

6. Hew, K.F., Cheung, W.S.: Use of three-dimensional (3-d) immersive virtual worlds in k-12 and higher education settings: A review of the research. British Journal of Educational Technology 41(1), 33-55 (2010)

7. Lockheed-Martin-Corp.: TO 1F-16AM-2-70JG-10-21 - Organizational Maintenance - Engine Removal and Installation - Model F100-PW-220/220E - USAF/EPAF Series - F-16/B Mid-Life Update aircraft, Technical Manual Job Guide. Lockheed Martin Corporation, Bethesda, MD, EUA (2009)

8. Morgado, L., Varajao, J., Coelho, D., Rodrigues, C., Sancin, C., Castello, V.: The attributes and advantages of virtual worlds for real world training. The Journal of Virtual Worlds and Education 1(1) (2010)

9. Nakasone, A., Prendinger, H., Holland, S., Hut, P., Makino, J., Miura, K.: Astrosim: Collaborative visualization of an astrophysics simulation in second life. IEEE Comput. Graph. Appl. 29, 69-81 (2009)

10. Orvis, K.A., Moore, J.C., Belanich, J., Murphy, J.S., Horn, D.B.: Are soldiers gamers? videogame usage among soldiers and implications for the effective use of serious videogames for military training. Military Psychology 22(2), 143 (2010)

11. Phillips, J., Berge, Z.L.: Second life for dental education. J. Dent. Educ. 73(11), 1260-1264 (2009)

12. Sotomayor, T.M.: Teaching tactical combat casualty care using the TC3 sim gamebased simulation: a study to measure training effectiveness. Stud. Health Technol. Inform. 154, 176-179 (2010); PMID: 20543293

13. Wiecha, J., Heyden, R., Sternthal, E., Merialdi, M.: Learning in a virtual world: Experience with using second life for medical education. Journal of Medical Internet Research 12(1) (January 2010)

14. Wyld, D.C.: A virtual explosion or snafu is lways better than a real one: Exploring the use of virtual worlds for simulation and training...and developing the leaders of tomorrow. In: Iskander, M., Kapila, V., Karim, M.A. (eds.) EIAT/IETA, pp. 73-78. Springer, Heidelberg (2008) 